\title{
Analytical model for charged polytropic stars with Van der Waals modified equation of state
}

\author{
Manuel Malaver \\ Universidad Marítima del Caribe, Departamento de Ciencias Básicas, Catia la Mar, Venezuela
}

Email address:

mmf.umc@gmail.com

\section{To cite this article:}

Manuel Malaver. Analytical Model for Charged Polytropic Stars with Van Der Waals Modified Equation of State. American Journal of Astronomy and Astrophysics. Vol. 1, No. 4, 2013, pp. 41-46. doi: 10.11648/j.ajaa.20130104.11

\begin{abstract}
We extend the work of Mafa Takisa and Maharaj (2013) by considering Van der Waals modified equation of state with polytropic exponent for anisotropic matter distribution in the study of a compact relativistic objects. New exact solutions for Einstein-Maxwell equations are generated in terms of elementary functions. The behaviour of physical variables as energy density, charge density and radial pressure is consistent with seminal treatments which suggest relevance in the description of relativistic compact stars.
\end{abstract}

Keywords: Compact Relativistic Objets, Van Der Waals Modified Equation of State, Energy Density, Radial Pressure

\section{Introduction}

One of the fundamental problems in the general theory of relativity is finding exact solutions of the Einstein field equations $[1,2]$. The resolution of Einstein's field equations has allowed to obtain exact solutions for relativistic stars and find mathematical appropriate models which allow to explain the nature of a neutron stars and the quasars [3]. The exact solutions of the system of Einstein-Maxwell's equations for gravitational fields with spherical symmetry are necessary to describe the physical properties of charged spheres and generated a great quantity of applications in stellar systems $[4,5]$.

In the production of the first theoretical models for relativistic stars are important the works of Schwarzschild [6], Tolman [7], Oppenheimer and Volkoff [8]. Schwarzschild [6] found analytical solutions that allowed to describe a star with uniform density, Tolman [7] developed a method to find solutions of static spheres of fluid and Oppenheimer and Volkoff [8] used Tolman's solutions to study the gravitational balance of neutron stars. It is important to mention Chandrasekhar's contributions [9] in the model production of white dwarfs in presence of relativistic effects and the works of Baade and Zwicky [10] who propose the concept of neutron stars and identify a astronomic dense objects known as supernovas.

Many researchers have used a great variety of mathematical techniques to try to obtain exact solutions for relativistic stars, since it has been demonstrated by
Komathiraj and Maharaj [4], Sharma et al. [5], Patel and Koppar [11], Tikekar and Singh [12], Feroze and Siddiqui [13], Malaver [14] and Mafa Takisa and Maharaj [15]. These analyses indicate that the system of equations Einstein-Maxwell is important in the description of astronomic dense objects.

Komathiraj and Maharaj [16], Malaver [17], Bombaci [18], Thirukkanesh and Maharaj [19], Dey et al [20] and Usov [21] assume linear equation of state for quark stars. Feroze and Siddiqui [13] consider a quadratic equation of state for the matter distribution and specify particular forms for the gravitational potential and electric field intensity. Mafa Takisa and Maharaj [15] obtained new exact solutions to the Einstein-Maxwell system of equations with a polytropic equation of state. Thirukkanesh and Ragel [22] have recently obtained particular models by specifying the polytropic index which are consistent with observations.

Considering the supposition of spherical symmetry and the existence of a conformal Killing vector, Mak and Harko [23] found a relativistic model of strange quark star. Esculpi and Alomá [24] for anisotropic relativistic charged matter by assuming the existence of a conformal Killing vector in static spherically symmetric spacetime.

In this paper we extend the work of Mafa Takisa and Maharaj [15] by considering of Van der Waals modified (VDWM) equation of state with polytropic index for the matter distribution where the gravitational potential and the electrical depend on adjustable parameters. The charge density is regular throughout the sphere and finite at the 
centre. Our objective is to find exact solutions to the Einstein-Maxwell system and propose a mathematical generalization of the results given in [14] in the study of compact relativistic objects. We have obtained new classes of spherical models of relativistic stars with polytropic index and all the results given in $[14,15]$ can also recovered as a particular case of our work.

This article is organized as follows, in Section 2, we present Einstein's field equations. In Section 3, there is chosen the form of the gravitational potential and the electrical field intensity that allows to solve the field

where $v(r)$ and $\lambda(r)$ are the two arbitrary functions. For charged perfect fluids the Einstein-Maxwell system of field equations are obtained as

$$
\begin{aligned}
& \frac{1}{r^{2}}\left(1-e^{-2 \lambda}\right)+\frac{2 \lambda^{\prime}}{r} e^{-2 \lambda}=\rho+\frac{1}{2} E^{2} \\
& -\frac{1}{r^{2}}\left(1-e^{-2 \lambda}\right)+\frac{2 v^{\prime}}{r} e^{-2 \lambda}=p-\frac{1}{2} E^{2} \\
& e^{-2 \lambda}\left(v^{\prime \prime}+v^{\prime 2}+\frac{v^{\prime}}{r}-v^{\prime} \lambda^{\prime}-\frac{\lambda^{\prime}}{r}\right)=p+\frac{1}{2} E^{2} \\
& \sigma=\frac{1}{r^{2}} e^{-\lambda\left(r^{2} E\right)^{\prime}}
\end{aligned}
$$

Using the transformations $x=c r^{2}, z(x)=e^{-2 \lambda(r)}$ and $A^{2} y^{2}(x)=e^{2 v(r)}$ where A and c are arbitrary constants, the Einstein-Maxwell system has the equivalent form

$$
\begin{gathered}
\frac{1-z}{x}-2 \dot{z}=\frac{\rho}{c}+\frac{1}{2 c} E^{2} \\
4 z \frac{\dot{y}}{y}-\frac{1-z}{x}=\frac{p_{r}}{c}-\frac{1}{2 c} E^{2} \\
4 x z \frac{\ddot{y}}{y}+(4 z+2 x \dot{z}) \frac{\dot{y}}{y}+\dot{z}=\frac{p_{t}}{c}+\frac{1}{2 c} E^{2} \\
\sigma^{2}=\frac{4 c z}{x}(x \dot{E}+E)^{2}
\end{gathered}
$$

$$
d s^{2}=-e^{2 v(r)} d t^{2}+e^{2 \lambda(r)} d r^{2}+r^{2}\left(d \theta^{2}+\sin ^{2} \theta d \varphi^{2}\right)
$$

equations as an equivalent set of differential equations using a transformations due to Durgapal and Bannerji. In Section 4, the physical features of the model are examined. Finally in Section 5, we conclude.

\section{The Field Equations}

Consider a spherically symmetric four dimensional spacetime whose line element is given in Schwarzschild coordinates by

$p_{r}, p_{t}, \rho, E$ and $\sigma$ are radial pressure, tangential pressure, energy density, electric field intensity and charge density, respectively and dots denote differentiation with respect to $\mathrm{x}$. In this paper, we assume the following Van der Waals modified equation of state

$$
p_{r}=\alpha \rho^{\Gamma+1}+\frac{\beta \rho^{\Gamma}}{1+\gamma \rho^{\Gamma}}
$$

where $\alpha, \beta$ and $\gamma$ are arbitrary constants and $\Gamma=1+$ $1 / \eta, \eta$ is the polytropic index.

\section{Polytropic Models with VDWM Equation of State}

Motivated by Mafa Takisa and Maharaj [15], Feroze and Siddiqui [13] and Thirukkanesh and Maharaj [19], we have chosen the form of the gravitational potential $\mathrm{z}$, which is well behaved and regular at the origin and the electric field intensity $\mathrm{E}$ is continuous, reaches a maximum and then it diminishes in the surface of the sphere. We make the specific choices

$$
\begin{gathered}
z=\frac{1+\beta x}{1+\alpha x} \\
\frac{E^{2}}{2 C}=\frac{\beta x}{(1+\alpha x)^{2}}
\end{gathered}
$$

On substituting (11) and (12) in (6) we obtain

$$
\frac{\rho}{C}=\frac{(\alpha-\beta)(3+\alpha x)-\beta x}{(1+\alpha x)^{2}}
$$

replacing (13) in (10), we have for the radial pressure

$$
p_{r}=\frac{\alpha[(\alpha-\beta)(3+\alpha x)-\beta x]^{\Gamma+1}}{(1+\alpha x)^{2(\Gamma+1)}}+\frac{\beta[(\alpha-\beta)(3+\alpha x)-\beta x]^{\Gamma}}{(1+\alpha x)^{2 \Gamma}+\gamma[(\alpha-\beta)(3+\alpha x)-\beta x]^{\Gamma}}
$$

On substituting (11), (12) and (14) in (7) we obtain 


$$
\frac{4(1+\beta x)}{(1+\alpha x)} \frac{\dot{y}}{y}-\frac{(\alpha-\beta)}{(1+\alpha x)}=\frac{\alpha[(\alpha-\beta)(3+\alpha x)-\beta x]^{\Gamma+1}}{C(1+\alpha x)^{2(\Gamma+1)}}+\frac{\beta[(\alpha-\beta)(3+\alpha x)-\beta x]^{\Gamma}}{C\left\{(1+\alpha x)^{2 \Gamma}+\gamma[(\alpha-\beta)(3+\alpha x)-\beta x]^{\Gamma}\right\}}-\frac{\beta x}{(1+\alpha x)^{2}}
$$

what is a linear equation in $\mathrm{y}$.

Now, using (12) in (9), the expression for the charge density is

$$
\frac{\sigma^{2}}{C^{2}}=\frac{2 \beta(1+\beta x)(\alpha x+3)^{2}}{(1+\alpha x)^{5}}
$$

The tangential pressure can be obtained from (8) with the help of (11), (12) and (15) and can be written as

$$
p_{t}=\frac{4 x C(1+\beta x)}{(1+\alpha x)} \frac{\ddot{y}}{y}+2 C\left[\frac{2+(\alpha+3 \beta) x+2 \alpha \beta x^{2}}{(1+\alpha x)^{2}}\right] \frac{\dot{y}}{y}-\frac{(\alpha-\beta) C+\beta C x}{(1+\alpha x)^{2}}
$$

The mass within a radius $r$ of the star is

$$
M(x)=\frac{1}{2} \int_{0}^{r} x^{2} \rho(x) d x
$$

The mass function is given for

$$
M(x)=\frac{6\left(\alpha \beta-\alpha^{2}-\beta\right)(1+\alpha x) \ln (1+\alpha x)+x^{3} \alpha^{3}\left(\alpha^{2}-\beta-\alpha \beta\right)+3\left(\alpha^{2}+\beta-\alpha \beta\right)\left(x^{2} \alpha^{2}+2 x \alpha\right)}{4 \alpha^{4}(1+\alpha x)}
$$

The following figures represent the graphs of $p_{r}, E^{2}$, $\rho, \sigma, \mathrm{M}$ and radial speed of sound $v_{s r}{ }^{2}$ with Van der Waals modified equation of state with polytropic index for some values of $\alpha, \beta$ and $\gamma$. In figures $1,2,3,4,5,6$ and 7 we generated the plots for the radial pressure, radial

$$
d s^{2}=-A^{2} y^{2}(x) d t^{2}+\frac{1+\alpha x}{(1+\beta x) 4 c x} d x^{2}+\frac{x}{c}\left(d \theta^{2}+\sin ^{2} \theta d \phi^{2}\right)
$$

When $\eta=1$, the equation of state (10) becomes

$$
p_{r}=\left(\frac{4}{15}\right) \rho^{3}+\frac{0.1 \rho^{2}}{1+0.1 \rho^{2}}
$$

If $\beta=0$ implies that $E=0$ and we find the uncharged polytropic model with $\eta=1$. Integrating (15) we obtain

$$
y=C_{1} \exp \left[\frac{a F(x)}{4}\right]
$$

Where $F(x)$ is

$$
F(x)=\frac{c a^{4} x^{5}+4 c a^{3} x^{4}+\left(6 c a^{2}-a^{5}\right) x^{3}+\left(4 c a-6 a^{4}\right) x^{2}+\left(c-13 a^{3}\right) x-10 a^{2}}{c(1+a x)^{4}}
$$

The corresponding line element is given by 


$$
d s^{2}=-A^{2} C_{1}^{2} \exp \left[\frac{a F(x)}{2}\right] d t^{2}+\frac{1+\alpha x}{4 c x} d x^{2}+\frac{x}{c}\left(d \theta^{2}+\sin ^{2} \theta d \varphi^{2}\right)
$$

The equation (24) is a new solution to the Einstein-Maxwell with polytropic index $\eta=1$.

For the uncharged model when $\eta=2$, the metric takes the form

$$
d s^{2}=-A^{2} C_{1}^{2} \exp \left[\frac{a F(x)}{96}\right] d t^{2}+\frac{1+\alpha x}{4 c x} d x^{2}+\frac{x}{c}\left(d \theta^{2}+\sin ^{2} \theta d \varphi^{2}\right)
$$

With $F(x)$ as

$$
\begin{aligned}
F(x)= & \left(\left(48 c x+144 a c x^{2}+144 a^{2} c x^{3}+48 a^{3} c x^{4}-\sqrt{2} \arctan h\left(\frac{1}{2 \sqrt{6+2 a x}}\right)\left[15 a^{3 / 2}+45 a^{5 / 2} x+45 a^{7 / 2} x^{2}+15 a^{9 / 2} x^{3}\right]\right.\right. \\
& -\sqrt{3+a x}\left[234 a^{3 / 2}+236 a^{5 / 2} x+66 a^{7 / 2} x^{2}\right]^{) /} c(1+a x)^{3}
\end{aligned}
$$

In Fig.1, the radial pressure is finite and decreasing for all the studied cases. In Fig. 2 , the condition $0 \leq v_{s r}^{2} \leq 1$ is maintained inside the stellar interior. In Fig. 3, the radial pressure is an increasing function with the energy density for each polytropic index. The electric field intensity reaches a maximum and then decreases, as it shows in Fig. 4. In Fig. 5 and 6, that represents energy density and charge density, respectively, we observe that are finite and monotonically decreasing functions. In Fig.7, the mass function is increasing function, continuos and finite.

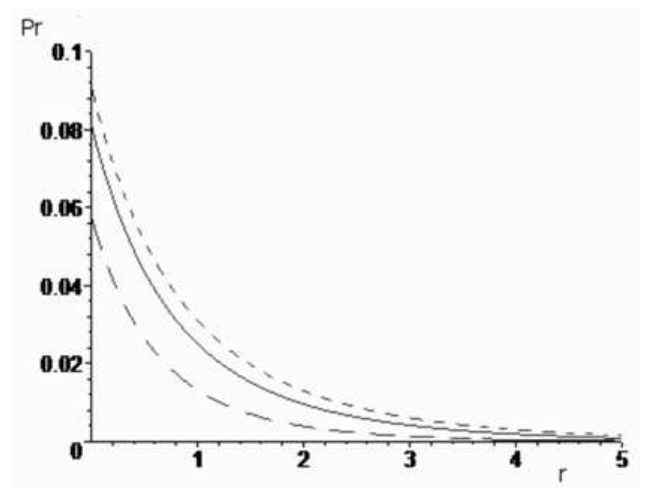

Fig 1. Radial pressure

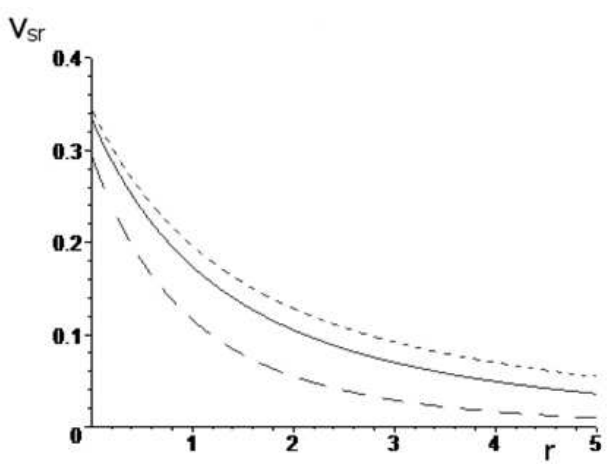

Fig 2. Radial speed of sound
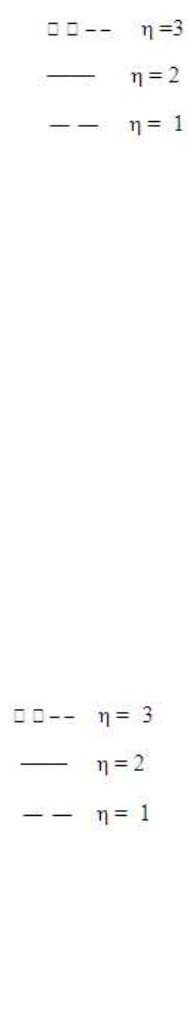

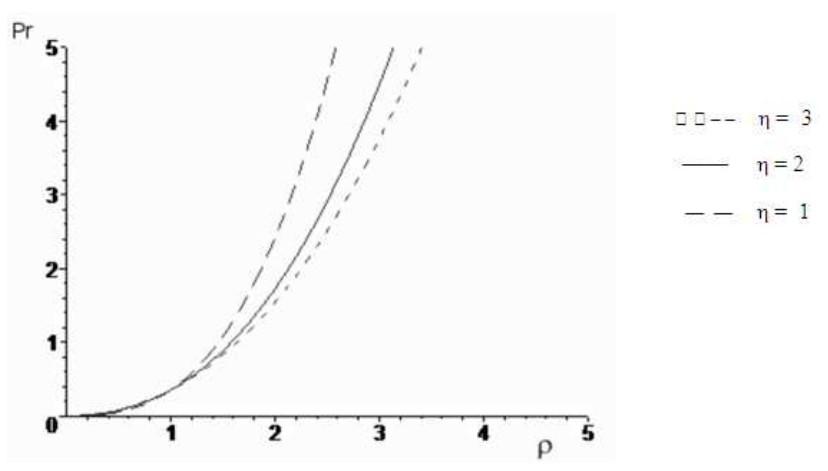

Fig 3. Radial pressure Pr vs energy density $\rho$

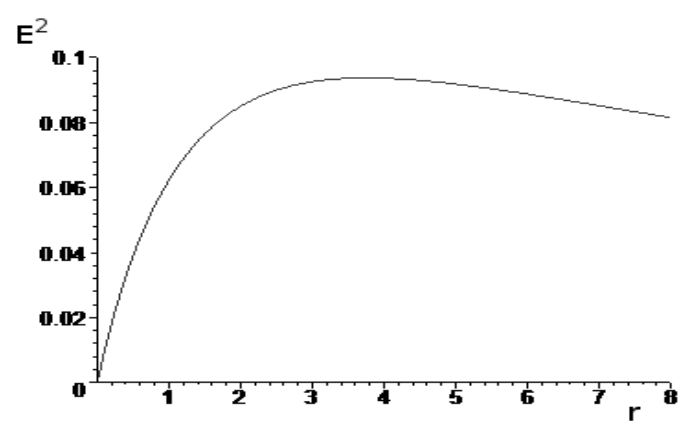

Fig 4. Electric field intensity

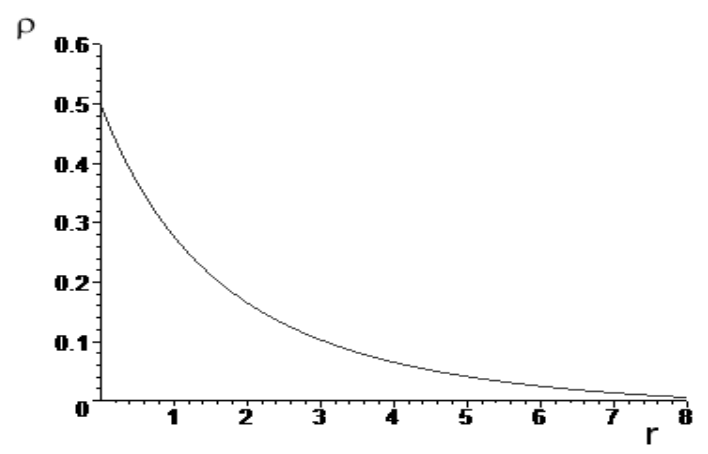

Fig 5. Energy density 


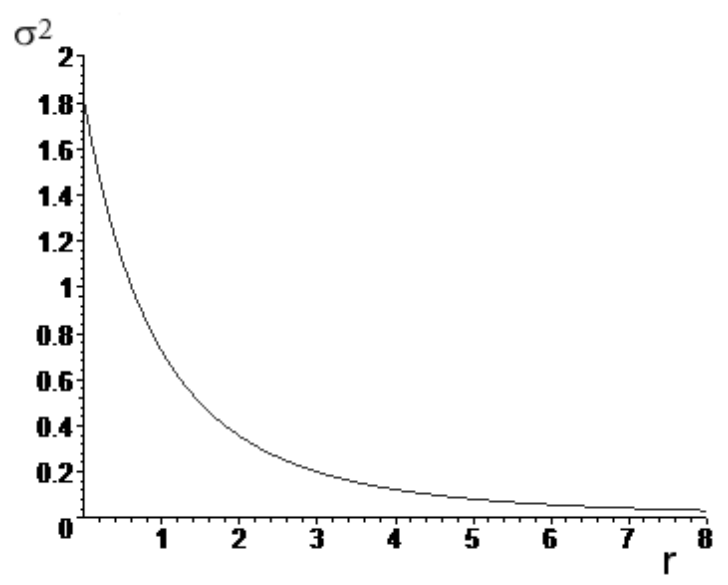

Fig 6. Charge density

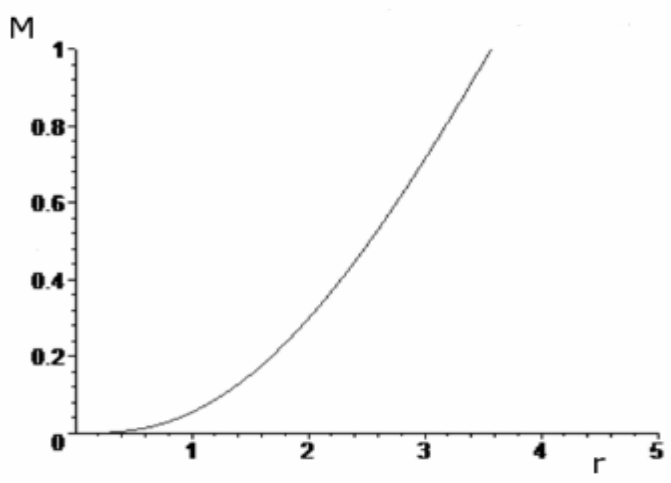

Fig 7. Mass

\section{Conclusion}

In this paper we have generated new exact solutions to the Einstein-Maxwell system considering Van der Waals modified equation of state with polytropic exponent. The new models presented in this paper may be used to model relativistic charged compact objects in astrophysics as strange and quark stars. Note that when the index $\eta \rightarrow \infty$, it recovered original Van der Waals modified equation of state that is $p_{r}=\alpha \rho^{2}+\frac{\beta \rho}{1+\gamma \rho}$ that corresponds to an isothermal sphere. For $\beta=0$, we found uncharged model with $\eta=1,2$ and some known solutions may be recovered for specific values of adjustable parameters. Note that the behaviour of radial pressures with and without polytropic index is essentially similar. However, an increase in polytropic term results in an growth of the values of the pressure in the new model.

Website: http://umc.academia.edu/ManuelMalaver

\section{References}

[1] P.K. Kuhfitting, "Some remarks on exact wormhole solutions", Adv. Stud. Theor. Phys., Vol. 5, pp. 365-367, June 2011.
[2] J. Bicak, "Einstein equations: exact solutions", Encyclopedia of Mathematical Physics, Vol. 2, pp.165-173, April 2006.

[3] M. Malaver, "Black Holes, Wormholes and Dark Energy Stars in General Relativity", Lambert Academic Publishing, Saarbrücken, 2013.

[4] K. Komathiraj and S.D.Maharaj, "Classes of exact EinsteinMaxwell solutions”, Gen. Rel.Grav., Vol. 39, pp. 2079-2093, August 2008.

[5] R. Sharma, S. Mukherjee and S.D. Maharaj, "General solution for a class of static charged stars", Gen.Rel. Grav., Vol. 33, pp. 999-110, June 2001.

[6] K. Schwarzschild, "Über das Gravitationsfeld einer Kugel aus inkompressibler Flüssigkeit”, Math.Phys.Tech, pp. 424434, 1916.

[7] R.C.Tolman, "Static Solutions of Einstein's Field Equations for Spheres of Fluid", Phys. Rev., Vol. 55, pp.364-373, february1939.

[8] J.R. Oppenheimer and G. Volkoff, "On massive neutron cores”, Phys. Rev., Vol.55, pp. 374-381, February 1939.

[9] S.Chandrasekhar, "Mass of Ideal White Dwarfs", Astrophys. J., Vol.74, pp. 81-82, January 1931.

[10] W.Baade and F. Zwicky, "Cosmic Rays from Super-Novae", Proc. Nat.Acad.Sci.U.S., Vol. (20), pp. 259-263, May 1934.

[11] L.K. Patel and S.K. Koppar, "A charged analogue of the Vaidya-Tikekar solution”, Aust. J. Phys, Vol 40, April 1987.

[12] R. Tikekar and G.P. Singh, "Gravitation and Cosmology", 4, 294.

[13] T. Feroze and A. Siddiqui, "Charged anisotropic matter with quadratic equation of state", Gen. Rel. Grav., Vol. 43, pp. 1025-1035, April 2011.

[14] M. Malaver, "Regular model for a quark star with Van der Waals modified equation of state", World Applied Programming., Vol. 3, pp. 309-313, July 2013.

[15] P.Mafa Takisa and S.D. Maharaj, "Some charged polytropic models", Gen.Rel.Grav., Vol. 45, pp. 1951-1969, October 2013.

[16] K. Komathiraj, K. and S.D. Maharaj, "Analytical models for quark stars”, Int.J.Mod. Phys., Vol. D16, pp. 1803-1811, November 2007.

[17] M. Malaver, "Análisis comparativo de algunos modelos analíticos para estrellas de quarks", Revista Integración, Vol. 27 , pp. 125-133, July 2009.

[18] I. Bombaci, "Observational evidence for strange matter in compact objects from the x- ray burster 4U 1820-30", Phys. Rev, Vol. C55, pp. 1587-1590, March 1997.

[19] S. Thirukkanesh and S.D. Maharaj, "Charged anisotropic matter with linear equation of state", Class. Quantum Gravity, Vol. 25, 235001, November 2008.

[20] M. Dey, I. Bombaci, J. Dey, S, Ray and B.C. Samanta, "Strange stars with realistic quark vector interaction and phenomenological density-dependent scalar potential ", Phys. Lett, Vol. B438, pp. 123-128, October 1998. 
[21] V.V. Usov, "Electric fields at the quark surface of strange stars in the color-flavor locked phase", Phys.Rev, Vol. D70, 067301 .

[22] S. Thirukkanesh and F.C. Ragel, "A class of exact strange quark star model”, Pramana J.Phys, Vol. 81, pp.275-286, August 2013.
[23] M.K. Mak and T. Harko, "Quark stars admitting a oneparameter group of conformal motions", Int.J.Mod.Phys, Vol. D13, pp. 149-156, January 2004.

[24] M. Esculpi and E, Alomá, "Conformal anisotropic relativistic charged fluid spheres with a linear equation of state", European Physical Journal, Vol. C67, pp. 521-532, June 2010. 